\title{
Experiencia de Diseño de Objeto Virtual de Aprendizaje OVA para Fortalecer el PEA en Estudiantes de Bachillerato
}

\author{
Design Experience of Virtual Learning Object OVA to Strengthen the PEA in High \\ School Students
}

Jorge Cristopher Delgado-Ramirez', Mayra Belén Tocto-Quezada² y Mayra Tatiana Acosta-Yela

\section{0 \\ EDICIÓN: ®E-CIVTAC}

Recibido: 15/julio/2020

Aceptado: 20/agosto/2020

Publicado: 25/septiembre/2020

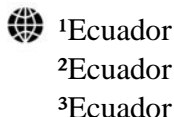

\section{IIIInstitución}

${ }^{1}$ Universidad Tecnica de Machala ${ }^{2}$ Universidad Técnica de Machala ${ }^{3}$ Universidad Técnica de Machala

\section{Correo Eletrónico}

1jdelgado@utmachala.edu.ec 2mtocto@utmachala.edu.ec ${ }^{3}$ macosta@utmachala.edu.ec

\section{DORCID}

1https://orcid.org/0000-0002-0123-4031 ${ }^{2}$ https://orcid.org/0000-0002-2541-5304 ${ }^{3}$ https://orcid.org/0000-0002-7774-8688

\section{Citar así: C4APA / IEEE}

Delgado-Ramirez, J., Tocto-Quezada, M. \& Acosta-Yela, M. (2020). Experiencia de Diseño de Objeto Virtual de Aprendizaje OVA para Fortalecer el PEA en Estudiantes de Bachillerato. Revista Tecnológica-Educativa Docentes 2.0, 9(2), 151-157.

https://doi.org/10.37843/rted.v9i2.158

J. Delgado-Ramirez, M. Tocto-Quezada y M. Acosta-Yela, "Experiencia de Diseño de Objeto Virtual de Aprendizaje OVA para Fortalecer el PEA en Estudiantes de Bachillerato", RTED, vol. 9, n. ${ }^{\circ} 2$, pp. 151157, sep. 2020.

\section{Resumen}

Los recursos tecnológicos han permitido la incursión de herramientas digitales interactivas o no interactivas en la educación, fortaleciendo el aprendizaje de los educandos en diferentes áreas del saber, no obstante, en el colegio "Dr. Juan Henríquez Coello", Ecuador, los estudiantes de bachillerato presentan falencias durante el proceso de enseñanza aprendizaje, en la asignatura de informática. Por tal motivo, el presente estudio, planteó como objetivo de investigación; el diseño de un Objeto Virtual de Aprendizaje para fortalecer los aprendizajes básicos imprescindibles de los estudiantes de bachillerato, siendo este un prototipo que surge como parte del proyecto COVA planteado en la Universidad Técnica de Machala. Dentro de la metodología se utilizó la investigación - acción participativa, además de utilizar como instrumentos de recolección de datos el cuestionario junto a una encuesta basada en escala de Likert permitiendo conocer el grado de satisfacción, además de la actitud de los 60 estudiantes de bachillerato quienes utilizaron el recurso digital. Entre los resultados, se logró evidenciar la propuesta del Objeto Virtual de Aprendizaje, donde los estudiantes demostraron una actitud positiva hacia el recurso propuesto obteniendo una aceptación del $90 \%$ por parte de los participantes. Por último, es necesario mencionar el uso de la metodología ADDIE como guía fundamental para la construcción e implementación de un Objeto Virtual de Aprendizaje permitiendo proponer al OVA como un recurso educativo digital para la interacción entre el docente - estudiante fortaleciendo el proceso de enseñanza aprendizaje de los contenidos de estudio de una asignatura.

Palabras clave: Objeto virtual de aprendizaje, recursos educacionales, material didáctico, innovación pedagógica.

\section{Abstract}

Technological resources have allowed the incursion of interactive or non-interactive digital tools in education, strengthening the learning of students in different areas of knowledge, however, at the "Dr. Juan Henríquez Coello", Ecuador, high school students have shortcomings during the teaching-learning process, in the computer science subject. For this reason, the present study, raised as research objective; the design of a Virtual Learning Object to strengthen the essential basic learning of high school students, this being a prototype that arises as part of the COVA project proposed at the Technical University of Machala. Within the methodology, participatory action research was used, in addition to using the questionnaire as data collection instruments together with a survey based on the Likert scale allowing to know the degree of satisfaction, as well as the attitude of the 60 high school students who they used the digital resource. Among the results, it was possible to show the proposal of the Virtual Learning Object, where the students demonstrated a positive attitude towards the proposed resource, obtaining an acceptance of $90 \%$ by the participants. Finally, it is necessary to mention the use of the ADDIE methodology as a fundamental guide for the construction and implementation of a Virtual Learning Object allowing to propose the OVA as a digital educational resource for the interaction between the teacher - student, strengthening the teaching-learning process of the study contents of a subject.

Keywords: Virtual learning object, educational resources, didactic material, pedagogical innovation. 


\section{Introducción}

Las tecnologías de la información y la comunicación (TIC) cumplen una función esencial en el sector académico de todos sus niveles, permitiendo que el entorno educativo evolucione favorablemente, aceptando los recursos tecnológicos e implementando estrategias metodológicas activas, así como el uso de técnicas y herramientas digitales que permitan el uso y creación de nuevos recursos digitales de aprendizaje los cuales pueden ser presentados y aplicados para su etapa de experimentación además de la validación del mismo, lo cual sería un aporte educativo en todos los niveles de estudio, teniendo en cuenta la forma e interacción de estos recursos de acuerdo a su edad, razonamiento o capacidades de los estudiantes.

El desconocimiento del manejo de herramientas $\mathrm{o}$ recursos digitales afectan principalmente a los docentes al momento de utilizar o proponer el uso de recursos digitales en las aulas de clase, más aún en asignaturas que cuentan con una infinidad de recursos digitales en la web, que pueden ser usados por él o los docentes de diferentes asignaturas permitiéndoles agilizar en tiempo la preparación y ejecución de las clases para así poder optimizar el proceso de enseñanza aprendizaje haciendo uso de diferentes recursos tecnológicos donde "las TIC permiten transferir y construir información, facilitando el aprendizaje, motivando al estudiante para asumir el conocimiento" (Mujica-Sequera, 2020, p. 41).

Así mismo es necesario conocer la definición de objetos de enseñanza donde Araya (2017), menciona que los objetos de enseñanza son todos los recursos que una persona puede utilizar en la organización y presentación de información dentro de los procesos de enseñanza donde el docente y estudiante cumplen su rol específico durante y después de la transmisión y adquisición de los contenidos de estudios en el entorno educativo.

A este contexto se debe mencionar también a los sistemas de gestión de aprendizaje o LMS (Learning Management Systems), los cuales según Moreno-Clari et ál. (2009), permiten el fortalecimiento de capacidades y habilidades de los sistemas educativos; de esta forma se plantea la creación de Objetos Virtuales de Aprendizajes (OVA), cuya finalidad es impulsar el crecimiento pedagógico, mediante el uso de herramientas tecnológicas las cuales permiten la difusión de conocimientos de forma interactiva e intuitiva, permitiendo una evaluación del contenido de forma divertida, evitando el sentimiento de temor de los estudiantes, esto permitirá determinar las debilidades y poder fortalecer dichos puntos frágiles.

\section{Objeto Virtual de Aprendizaje}

De acuerdo con Rengifo et ál. (2015), un (OVA) puede ser considerado de gran importancia dentro del proceso educativo, pues se estructura de varios componentes internos como: contenidos, actividades de aprendizaje y elementos de contextualización permitiendo contribuir al desarrollo de esquemas virtuales de aprendizaje, así mismo potenciar la creatividad de la comunidad educativa ayudando de esta forma al proceso de adquisición de nuevos conocimientos.

A esto se complementa lo que mencionan (García \& Acevedo, 2016), quienes afirman que durante varios años la tecnología ha dado paso a la evolución de nuevos recursos digitales, lo cual ha permitido una mejora en el ámbito educativo como ayuda en el desarrollo de un aprendizaje constructivo, haciendo uso de tecnologías emergentes que propician en la actualidad a generar sus propios contenidos.

A este punto se debe mencionar refiriendo a Sharp (2019), un OVA ha abarcado la utilización de diferentes dispositivos tecnológicos, como lo es el uso del celular o Tablet permitiendo al estudiante una adaptación a la falta de herramientas tecnológicas como seria el computador de escritorio o computador portátil, posibilitando un buen aprovechamiento de estas herramientas en los procesos de enseñanza aprendizaje en un entorno educativo.

De acuerdo con Vargas (2018), desde su perspectiva un OVA es un recurso muy importante en el proceso educativo, permitiendo utilizarlo como un repositorio de contenidos basándose en las necesidades educativas de la institución educativa, siendo de mucha utilidad antes, durante y después de las jornadas educativas planteadas en los diferentes espacios de aprendizaje de las instituciones educativas.

Partiendo de estas primicias se puede establecer la utilización de un OVA en la educación como un material didáctico de enseñanza aprendizaje, posibilitado la incorporación de nuevos recursos 
educativos digitales en la aplicación de metodologías tradicionales o activas dentro de los procesos de enseñanza aprendizaje, fomentando el uso de recursos tecnológicos educativos los cuales serían considerados por los docentes como un instrumento de ayuda en el proceso de enseñanza aprendizaje en el entorno educativo.

\section{Diseñando un Objeto Virtual de Aprendizaje}

La elaboración y diseño del OVA se basó en la metodología ADDIE, según Preciado (2013), la metodología consiste en una guía descriptiva para la construcción de herramientas de formación y apoyo, a su vez puede ser fácilmente aplicado en diferentes áreas educativas asegurando una gestión del conocimiento establecido en objetivos determinados, la cual es caracterizada por poseer un esquema de información de fácil acceso y manejo, este modelo cuenta con 5 fases; Análisis, Diseño, Desarrollo, Implementación y Evaluación.

Análisis. Dentro de la fase de análisis se debe iniciar realizando el estudio del problema planteado en la investigación, donde se puede definir principalmente que aspectos deben considerarse para que el OVA pueda alinearse a la dinámica educativa lo cual considere un valor agregado a la búsqueda de aprendizajes, habilidades y conocimientos que se desee obtener en el entorno educativo.

Diseño. En esta etapa una vez realizado el análisis del problema se busca diseñar una estructura que se acople al proceso de enseñanza aprendizaje, para lo cual se diseña inicialmente un bosquejo de cómo se piensa realizar el diseño del producto en sus primeras ideas, lo cual conlleva un proceso de elaboración de diferentes borradores antes de poder llegar a obtener lo que se presenta en la Figura. 1, Figura.2, considerando la variación de los diseños de acuerdo con la necesidad de la comunidad educativa.

\section{Figura 1}

Boceto de portada del recurso de OVA.

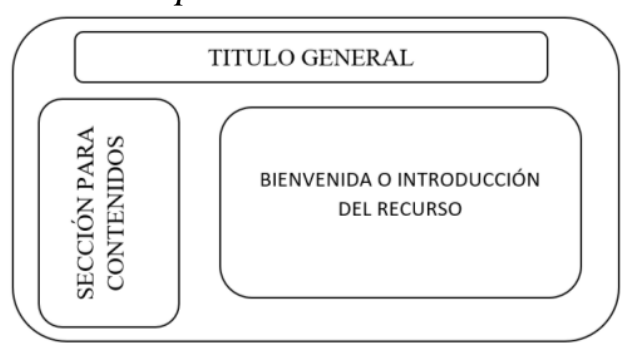

Nota. La figura representa la portada del OVA, en este caso la primera interfaz inicial, elaborado por los autores.

\section{Figura 2}

Boceto de secciones de trabajo del recurso de OVA.

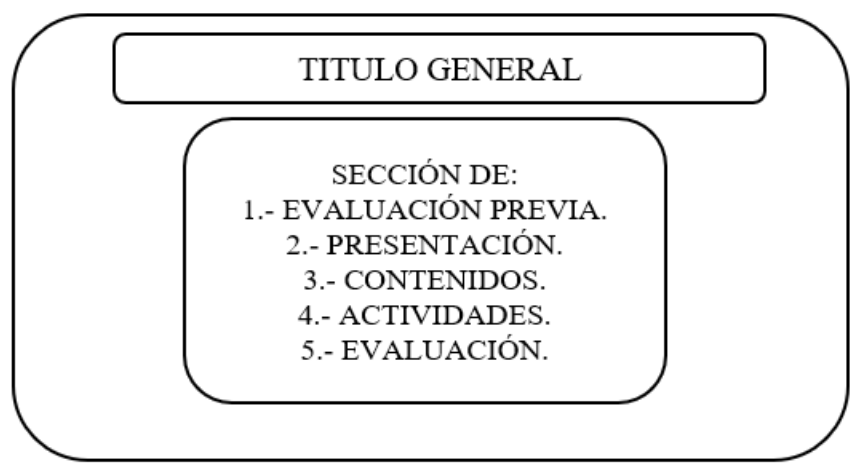

Nota. La figura representa la interfaz de secciones que contiene el OVA, elaborado por los autores.

En este punto es necesario destacar la importancia de la elaboración de un bosquejo inicial el cual permite tener un punto de partida para poder realizar la búsqueda y estudio de diferentes programas como lo muestra la Tabla1, posibilitando obtener una comparativa informativa y de sustento para la elaboración del recurso, permitiendo de este modo poder establecer el diseño y programa base de donde se realizará el OVA.

\section{Tabla 1}

Lista de recursos web relacionados al diseño del OVA.

\begin{tabular}{|c|c|c|}
\hline Dirección web & Institución & Contenidos \\
\hline https://lvera3.wixsite.com/tftmmiii & $\begin{array}{l}\text { Universidad } \\
\text { de Mendoza }\end{array}$ & $\begin{array}{l}\text { Violencia } \\
\text { Interpersonal }\end{array}$ \\
\hline http://danielgarci6.wix.com/sr_garcia_macaco & $\begin{array}{l}\text { Ágora } \\
\text { Portals } \\
\text { International } \\
\text { School }\end{array}$ & $\begin{array}{l}\text { PLE en el } \\
\text { aula: } \\
\text { Historias } \\
\text { sobre tutoría } \\
\text { en el aula. }\end{array}$ \\
\hline https://competencialectora.wixsite.com/itmc & $\begin{array}{l}\text { Universidad } \\
\text { Privada } \\
\text { Norbert } \\
\text { Wiener }\end{array}$ & $\begin{array}{l}\text { Uso de una } \\
\text { página web } \\
\text { en Wix para } \\
\text { fortalecer la } \\
\text { competencia } \\
\text { comunicativa } \\
\text { lectora. }\end{array}$ \\
\hline
\end{tabular}

Nota. La figura nos presenta un listado de enlaces de OVA de los cuales se toman como base, elaborado por los autores.

Desarrollo. Dentro del proceso de desarrollo se consideró como programa base para el diseñó del OVA a la plataforma de creación de páginas web gratuita WIX (http://es.wix.com), siendo esta una plataforma de creación de sitios web, considerada a su vez un editor online para la creación y publicación de sitios web de manera sencilla en flash, además permite realizar un indexado en buscadores, utiliza como lenguaje base el HTML 5, además de mencionar 
una de sus características es poseer una interfaz amigable al momento de programar sus funciones, asimismo teniendo en cuenta a Cañizares (2013), quien expresa: "Wix es una herramienta muy útil para la educación, ya que permite agrupar todo tipo de recursos en distintos soportes dentro de una misma web" (p. 69).

Una vez seleccionado el programa y teniendo en cuenta el bosquejo de la Figura. 1 se inició a elaborar el recurso revisando de forma general las herramientas que presenta la plataforma WIX, en cuanto a su diseño se tomó en cuenta criterios de Raposo y Ruiz (2005) donde mencionan que se debe respetar los principios de simplicidad, la coherencia, la claridad, la estética, el uso del espacio en blanco y el tiempo. Luego de varias propuestas de diseño se decidió por las presentaciones que se muestran en la Figura. 3 y Figura. 4.

\section{Figura 3}

Diseño de presentación inicial del objeto virtual de aprendizaje.

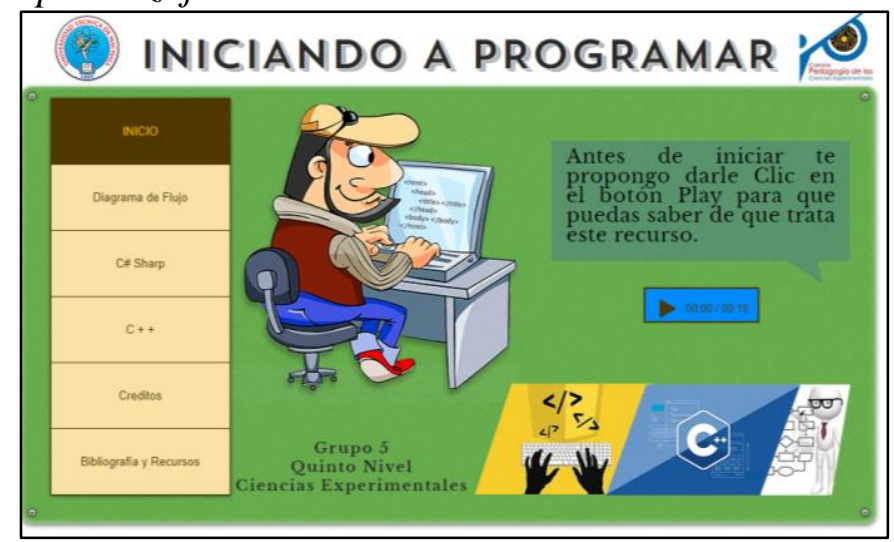

Nota. Se presenta la primera interfaz inicial que observará el usuario del OVA, elaborado por los autores.

\section{Figura 4}

Diseño de estructura de actividades del OVA.

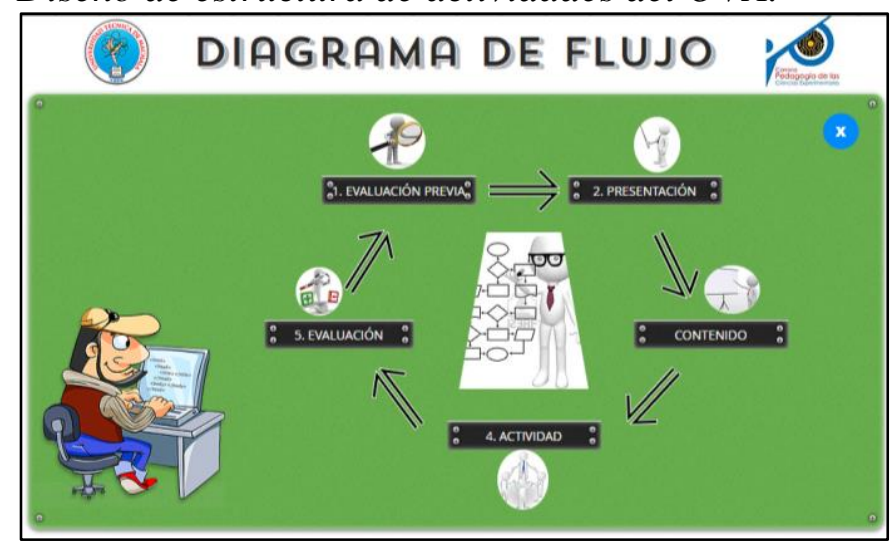

Nota. Se presenta la sección del tema de Diagrama de fujo que contiene el OVA, elaborado por los autores.
Implementación. La etapa de implementación tuvo lugar en el Colegio de bachillerato "Dr. Juan Henríquez Coello" dando a conocer inicialmente el recurso OVA a los estudiantes de bachillerato presentando las respectivas indicaciones de uso del recurso por considerarse un nuevo material didáctico dentro de la asignatura y por lo tanto desconocido por los estudiantes a quienes necesariamente correspondía realizar la socialización del recurso, lo cual se evidencia en la Figura. 5 y Figura. 6.

\section{Figura 5}

Docente de la asignatura de informática.

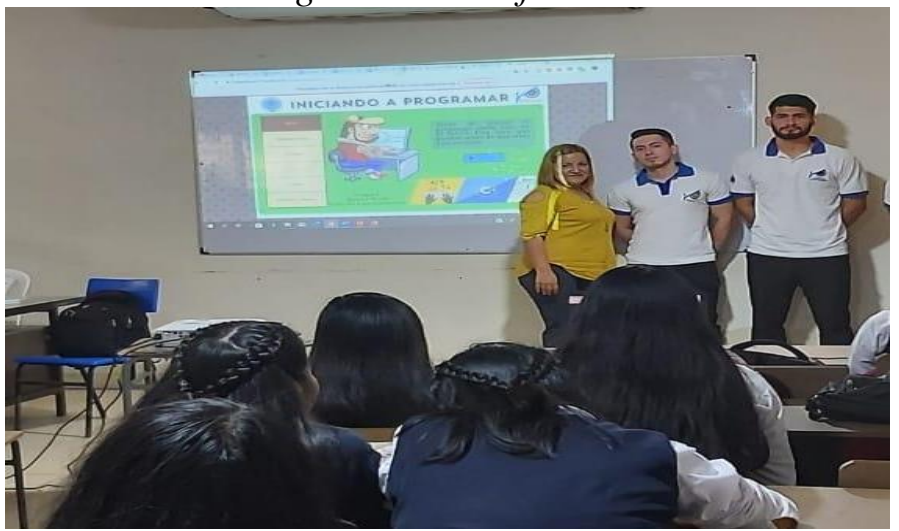

Nota. Socialización del OVA con docente de la asignatura de informática y estudiantes de bachillerato, elaborado por los autores.

\section{Figura 6}

Estudiantes de Bachillerato.

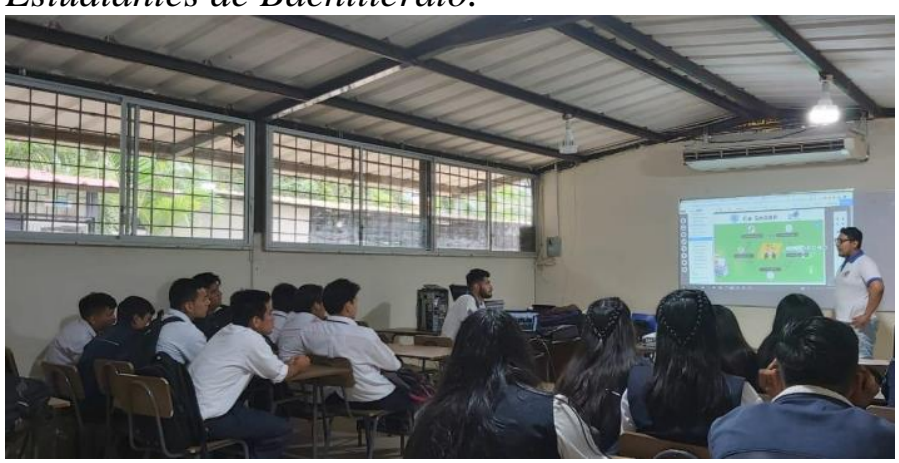

Nota. Aplicación del OVA con estudiantes de bachillerato, elaborado por los autores.

Evaluación. Antes de implementar el recurso tecnológico es necesario la evaluación previa de su funcionamiento con el fin de poder considera los posibles problemas al momento de ser utilizado por parte de los estudiantes, en esta etapa es necesario tener en cuenta "el estudio de las herramientas para su respectivo mejoramiento se constituye en el principio natural de la tecnología" (Jiménez, 2007, p. 74). 
Por tal motivo la evaluación previa del recurso se desarrolló con un grupo de estudiantes de la carrera de Pedagogía de las Ciencias Experimentales de la Facultad de Ciencias Sociales de la Universidad Técnica de Machala logrando verificar la funcionalidad del recurso generando las observaciones necesarias desde el punto de vista de estudiante pudiendo realizar las mejoras necesarias propuestas antes y después de su implementación.

\section{Metodología}

El desarrollo de la Investigación se aplicó la Investigación Acción Participativa (IAP), la cual permite obtener información a través del proceso de intervención y participación de los sujetos en el lugar de estudio. Según (Zapata \& Rondán, 2016) indica que "la IAP es en realidad una amplia familia que incluye diferentes maneras de hacer investigación proyectando el cambio social con la participación de la gente y que ha tenido diversos desarrollos en el mundo" (p. 7).

La IAP cumple con las Fases de Diagnóstico, Programación, Conclusiones y Propuestas.

(Marti, 2017) Indica y explica las Fases "Diagnóstico. Conocimiento contextual del territorio y acercamiento a la problemática (...). Programación. Proceso de apertura a todos los conocimientos y puntos de vista existentes, utilizando métodos cualitativos y participativos (...). Conclusiones y propuestas. Negociación y elaboración de propuestas concretas" (p. 2).

Existe una Pre-Investigación denominada Síntomas, demanda y elaboración del proyecto: Es aquí en donde determinan los sujetos involucrados en el desarrollo de la Investigación, así como los síntomas de esta y la delimitación del problema En la Primera Etapa denominada Diagnóstico se realiza un acercamiento a la problemática, a través de técnicas que permitan dicho acercamiento.

Durante el proceso de obtención de información se aplicaron técnicas cualitativas y cuantitativas las cuales permitieron observar la realidad de la situación en el lugar de estudio; siendo la Ficha FODA o DAFO una de las técnicas aplicadas y la que permitió la obtención de información sobre las fortalezas, oportunidades, debilidades y amenazas del entorno educativo, como se muestra en el extracto de la Figura. 7.

\section{Figura 7}

Extracto de información de la ficha FODA.

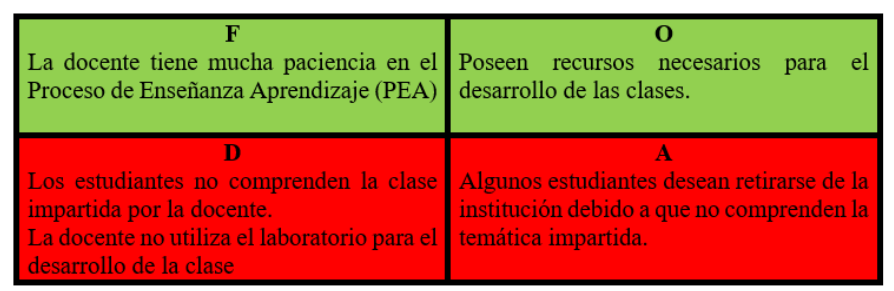

Nota. Información recogida en la observación mediante ficha FODA, elaborado por los autores.

La información recopilada de la ficha FODA permitió identificar la necesidad de implementar un recurso extra dentro del proceso de enseñanza aprendizaje en la asignatura de informática teniendo en cuenta el lugar de uso del recurso podía ser dentro o fuera de las aulas de clase con la finalidad de poder a la información de forma interesante y divertida, así como también fortalecer diferentes temas considerados significativos en el aprendizaje del educando.

\section{Resultados}

Se aplicaron encuestas antes y después de la implementación del OVA, con la finalidad de observar la aceptación y la motivación por el aprendizaje a través de este. Estas técnicas fueron basadas en la escala de Likert, permitiendo medir la intensidad del sentimiento del estudiante a través de los resultados, los cuales podrían haber sido a favor o en contra. Basándose en esta información se logró receptar lo detallado en la Tabla 2.

\section{Tabla 2.}

Comparación de Resultados en la Implementación del OVA

\begin{tabular}{lcccc}
\hline \multicolumn{1}{c}{ ANTES DEL OVA } & \multicolumn{3}{c}{ DESPUÉS DEL OVA } \\
\hline $\begin{array}{l}\text { ¿Considera necesario la implementación de } \\
\text { un OVA dentro de su proceso de formación } \\
\text { académica? }\end{array}$ & $\begin{array}{l}\text { Según su criterio: iSiente que es } \\
\text { necesario la implementación de un OVA } \\
\text { dentro del proceso de formación } \\
\text { académica en la asignatura? }\end{array}$ \\
\hline $\begin{array}{l}\text { Muy } \\
\text { importante }\end{array}$ & 16 & $27 \%$ & 44 & $73 \%$ \\
\hline $\begin{array}{l}\text { Importante } \\
\text { Neutral }\end{array}$ & 26 & $42 \%$ & 11 & $19 \%$ \\
\hline $\begin{array}{l}\text { Poco } \\
\text { importante }\end{array}$ & 0 & $0 \%$ & 0 & $8 \%$ \\
\hline $\begin{array}{l}\text { No es } \\
\text { importante }\end{array}$ & 0 & $0 \%$ & 0 & $0 \%$ \\
\hline Total & 60 & $100 \%$ & 60 & $100 \%$ \\
\hline
\end{tabular}

Nota. Resultados al implementar el OVA, elaborado propia. 


\section{Figura 8}

Resultado de encuesta antes de la implementación del OVA.

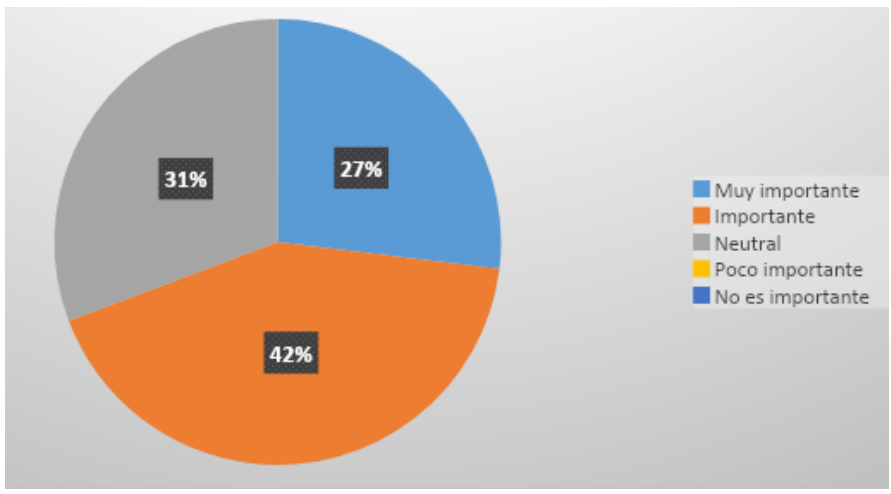

Nota. Gráfico estadístico que refleja las respuestas antes de la implementación del OVA, elaborado por los autores.

\section{Figura 9}

Resultado de encuesta después de la implementación del OVA

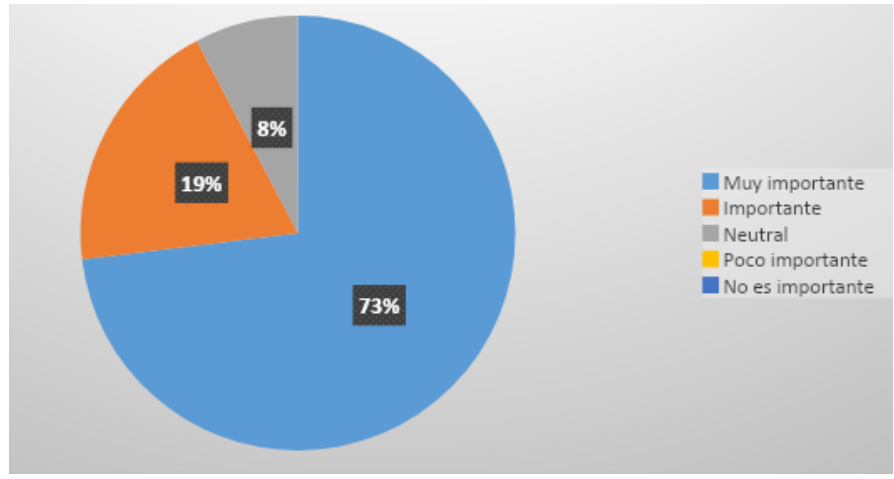

Nota. Gráfico estadístico que refleja las respuestas antes de la implementación del OVA, elaborado por los autores.

Dentro de los resultados emitidos por los 60 participantes es evidente su perspectiva en el uso del recurso OVA, pudiendo hacer referencia qué en primera instancia los estudiantes no tenían total seguridad de la importancia del uso de recursos digitales, por tal motivo luego de la implementación del recurso educativo digital los estudiantes en su mayoría estuvieron de acuerdo con el uso del recurso dentro de la asignatura de informática.

La Etapa de Diagnóstico y Programación de la investigación se encuentran muy ligadas a las etapas de la metodología ADDIE dentro de la construcción del OVA. Así mismo se puede evidenciar, mediante la aplicación del OVA y a través de las respuestas obtenidas por los estudiantes en la encuesta, referente al grado de satisfacción al momento de implementar un OVA en el proceso de enseñanza aprendizaje tal como se muestra en la Tabla 3 y Figura. 10.

\section{Tabla 3}

Respuestas sobre el grado de satisfacción de la Implementación de OVA en clase.

\begin{tabular}{ccc}
\hline Valor & Frecuencia & Porcentaje \\
\hline Extremadamente satisfecho & 37 & $62 \%$ \\
\hline Muy satisfecho & 14 & $23 \%$ \\
\hline Moderadamente satisfecho & 9 & $15 \%$ \\
\hline Poco satisfecho & 0 & $0 \%$ \\
\hline No satisfecho & 0 & $0 \%$ \\
\hline Total & $\mathbf{6 0}$ & $\mathbf{1 0 0 \%}$ \\
\hline
\end{tabular}

Nota. Valoración de los estudiantes según el grado de satisfacción luego de la implementación del OVA, elaborados por los autores.

\section{Figura 10}

Resultado de grado de satisfacción de la Implementación de OVA

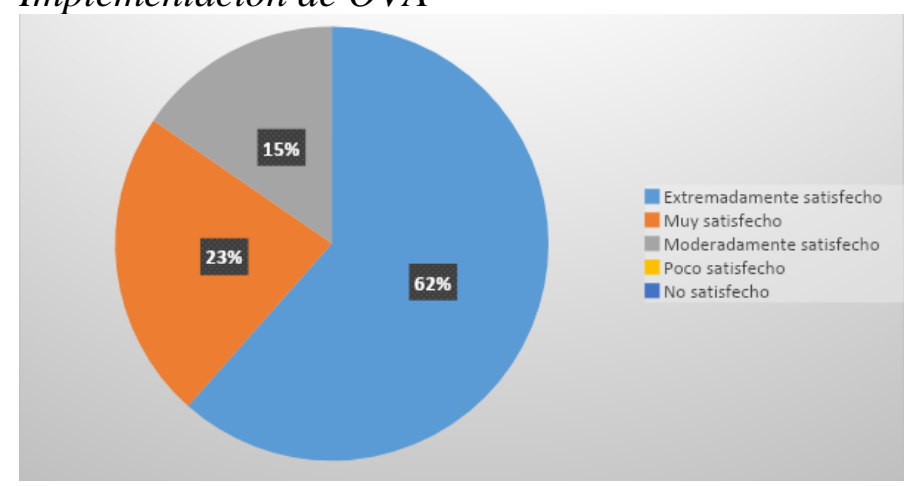

Nota. Gráfico estadístico referente al grado de satisfacción de los participantes en la implementación del OVA, elaborado por los autores.

\section{Conclusiones}

En este estudio, se intentó obtener un grado de madurez significativo en cuanto a la propuesta de elaboración y diseño del proyecto OVA, generando la participación conjunta de todos los involucrados en el desarrollo del producto final obteniendo comentarios positivos por los docentes de la institución donde se implementó el OVA, permitiendo así fortalecer la vinculación que existe entre la institución de educación superior y la institución de educación media del Ecuador.

El diseño del objeto virtual de aprendizaje despertó el interés por los docentes de otras asignaturas entre ellas matemáticas y biología quienes mencionaron a través de comentarios en plenaria de docentes la importancia de este tipo de recursos digitales pueden aportar significativamente al proceso educativo de las instituciones educativas fortaleciendo los vacíos producidos por la brecha digital aún existente en gran parte de los docentes 
quienes presentan debilidades en el uso de recursos digitales en el entorno educativo.

Se debe destacar la importancia del producto de este estudio es uno de los resultados obtenidos de los procesos de vinculación sostenidos entre la Universidad Técnica de Machala y el Distrito educativo 07D02 el cual permite a la Carrera de Pedagogía de las Ciencias Experimentales de la Facultad de Ciencias Sociales de la Universidad Técnica de Machala poder desarrollar e implementar diferentes proyectos de investigación en el campo de la educación.

\section{Referencias}

Alayón, P. C. (2017). Una experiencia de estandarización utilizando el modelo ADDIE en la elaboración de guías temáticas. e-Ciencias de la Información, 7(1), 1-14.

Araya, A. M. (2017). Integración de recursos audiovisuales y multimedia como objetos de aprendizaje en escuelas públicas de Costa Rica con acceso a computadoras XO. Actualidades Investigativas en Educación, 17(1).

Cañizares, M. (2013). Wix en el aula. Aula de Innovación Educativa, 220, 69-70.

Castro, V., Washington, J., \& Santos Jiménez, O. C. (2019). Las tecnologías del aprendizaje y el conocimiento en la formación docente. Conrado, 15(68), 180-186.

Francés García, F. J., Alaminos Chica, A., Penalva Verdú, C., \& Santacreu Fernández, O. A. (2015). La investigación participativa: métodos y técnicas. Universidad de Cuenca.

García Carmona, A., \& Acevedo, J. A. (2016). Una controversia de la Historia de la Tecnología para aprender sobre Naturaleza de la Tecnología: Tesla vs. Edison-La guerra de las corrientes. Enseñanza de las ciencias: revista de investigación y experiencias didácticas, 34 (1), 193-209.

Garcia, D.J. (2013). PLE en el aula: historias sobre tutorías en secundaria. En L. Castaneda y J. Adell (Eds.), Entornos Personales de Aprendizaje: Claves para el ecosistema educativo en red (p. 117-121). Alcoy: Marfil.

Martí, J. (2017). La investigación-acción participativa: estructura y fases. Recuperado de: http://www.redcimas.org/wordpress/wpcontent/uploads/2012/08/m_JMarti_IAPFASES.pdf

Moreno-Clari, P., Roig-García, D., \& López-Bueno, A. (2009). Sistema de gestion del aprendizaje (LMS) integrado para su uso en educacion superior. RISTI (Revista Iberica de Sistemas $e$ Tecnologias de Informacao), (3), 39-53.

Ponce, R. E. V. (2015). Wix para la creación de sitios web. Parte II. Doctoral disertación, Universidad Nacional de San Luis.

Preciado, V. P. (2013). La gestión del conocimiento mediante el modelo ADDIE y su aplicación en un caso de logística. Scopus, 30 .
Rengifo, P., Yois, S., Jaramillo Morales, C. O., \& Verástegui González, F. A. (2015). Desarrollo de objetos virtuales de aprendizaje como estrategia para fomentar la permanencia estudiantil en la educación superior. Revista EAN, (79), 116-129.

Rivas, M. R., \& Ruiz, M. I. D. (2005). Practicando con TICs en contextos formativos. Universidad de Vigo.

Sharp, J. (2019). Diseño, desarrollo y creación de contenido para un sitio web de educación abierta Física para MRT Education. Scopus, 15

Solano Peréz, S. L. (2018). Uso de una página web en Wix para fortalecer la competencia comunicativa lectora en los estudiantes del grado noveno del Instituto Técnico Industrial Monseñor Carlos Ardila García.

Vargas, J. (2018). Comunicación de vías profesionales a través de los sitios web del programa de grado asociado: una evaluación de referencia. Scopus, 10.

Vera, L. A. (2016). Sitio web interactivo para el aprendizaje del tema" Violencia interpersonal" correspondiente a la asignatura Psicología Social (Master's thesis)

Zapata, F., \& Rondán, V. (2016). La investigación-acción participativa. Recuperado de: http://mountain.pe/recursos/attachments/article/168/Investigaci on-Accion-Participativa-IAP-Zapata-y-Rondan.pdf

Mujica-Sequera, R. (2020). E-Learning como estrategia pedagógica en la educación superior. Revista Tecnológica-Educativa Docentes 2.0, 9(1), 37-41. https://doi.org/10.37843/rted.v9i1.103

Jiménez, D. F. L. (2007). La naturaleza de las tecnologías de información y comunicación: las TIC como determinantes de la organización y de la sociedad de la información. Palabra Clave, $10(1)$ 